\title{
A New Technique for Characterising Planetary Nebulae in External Galaxies
}

\author{
K. Taylor and N.G. Douglas 1 \\ Anglo-Australian Observatory, Sydney, Australia
}

\begin{abstract}
Planetary Nebulae are proving to be powerful tertiary distance indicators as well as sensitive probes of a galaxy's velocity field and mass distribution. We present a new technique for determining the positions, luminosities and radial velocities of the PNe in an external galaxy which may enable a one-step measurement of all these parameters.
\end{abstract}

\section{Introduction}

Planetary Nebulae ( $\mathrm{PNe}$ ) in external galaxies provide a powerful tertiary distance indication through their well behaved luminosity function (Jacoby, G.H., Ciardullo, R. \& Ford H.C. 1990 Ap. J. 365, p. 332). Being very narrow-line $\left(\leq 60 \mathrm{~km} \cdot \mathrm{s}^{-1}\right)$, almost pure emission-line objects, they also provide a unique opportunity to study the dynamics of early-type galaxies out to isophotal radii well beyond any other technique presently available. For example, Ford et al (Ford, H.C., Ciardullo, R., Jacoby, G.H. \& Hui, X. 1989. IAU Symposium 131, p. 335) probed the kinematics of the spheroid of Cen $A$ out to a distance to $20 \mathrm{kpc}$, equivalent to $\sim 4$ effective radii $\left(R_{e}\right)$.

Until now, measurement of the PNe themselves has been a two-step process: identification in CCD frames taken through interference filters, followed up by single object or fibre spectroscopy. Many of the pioneers in this field are putting their hopes in the HST to extend the use of PNe, especially in distance determination, and hope to get satisfactory results out to the Virgo cluster. We propose a variant of the traditional approach to the discovery of PNe in external galaxies which promises to be a powerful adjunct to this work.

\section{Present Techniques}

Traditionally, the emission-line spectrum of PNe has been used to distinguish them from the background sky and stellar continuum. The [OIII] $\lambda 5007$ line is generally the strongest and has a wavelength which is well placed for CCD observations. The strategy has been to take on- and off-band images through interference filters to look for candidate PNe. This method suffers from a number of problems, however:

\footnotetext{
${ }^{1}$ Permanent Address: Kapteyn Astronomical Institute, Groningen, The Netherlands
} 
- Obtaining reliable fluxes from narrow-band images is fraught with difficulties. In principle the temperature dependence of the interference filter and the detailed shape of its bandpass as a function of field position, and the radial velocity of the detected PNe, must be known before accurate photometry can be achieved. In practice, simple flat fielding is used in the hope that these effects are not dominant. For similar reasons, continuum subtraction of the off-band image is also unreliable.

- Variations in seeing and source position between the on- and off-band images degrade the sensitivity of the technique.

- For optimum contrast against the sky and galaxy background continuum, the on-band interference filter bandwidth should be matched to the velocity dispersion of the galaxy $(\sim 10-15 \AA)$. For safety's sake however the bandpass of the filter is usually made large enough $(\sim 30-40 \AA)$ that variations in radial velocity of the PNe do not induce significant throughput variations within the filter.

- Follow-up fibre spectroscopy is needed to determine the PNe kinematics, and this demands very accurate astrometry. The logistics of locating and then using the PNe for dynamical studies may take two seasons of observations.

An alternative technique has been investigated by $\mathrm{K}$. Freeman and coworkers using slitless imaging through a spectrograph (priv. comm. 1990). Given the same on-band interference filter and instrumental efficiency, the discrimination between continuum background and emission-line source is the same as with direct narrow-band imaging. The $\mathrm{PNe}$ simply appears as a seeing-disk limited emission spike above a dispersed continuum. The position of the spike is a function of both location in the field and radial velocity about the galaxy and hence some other method has to be employed to separate these two parameters. The method used has been to combine the slitless image with a direct image (sometimes taken through the spectrograph by replacing the grating with a mirror). This, however, still has many of the problems of direct imaging noted earlier.

\section{Novel Principle}

A simple, but apparently unexploited, solution to this problem is to take two slitless images with the spectrograph rotated through $180^{\circ}$ in between (in principle any angle will do). Given a double detection, one is then able to recover both the location and velocity of each PNe. Since the continuum is expected to vary slowly with wavelength compared with the emission lines, detection should be straightforward. Indeed, by differencing the two images the background continuum will, to a certain approximation, be nulled. It seems to us that detection sensitivity might be limited only by the shot-noise in the background, systematic effects being reduced to a minimum.

The technique is also very robust in regard to fluxing, since stars in the observed field readily supply the bandpass response of the filter enabling the flux of each PNe to be calibrated without the uncertainties inherent in on- and off-band imaging. This allows us to match the bandpass of the interference filter to a nominal maximum galaxy velocity dispersion, reducing background continuum contamination by a large factor. 


\section{Practical realisation}

We consider a simple, slitless adaption of the existing cassegrain spectrograph on the AAT (see Fig 1). The spectrograph has a $150 \mathrm{~mm}$ collimated beam and a camera of focal length $250 \mathrm{~mm}$. Using a $1200 \mathrm{~g} / \mathrm{mm}$ grating in second order with a CCD having $24 \mu \mathrm{m}$ pixels, a $1^{\prime \prime}$ monochromatic image has a wavelength spread of $\sim 3.3$ pixels giving a wavelength resolution of $\sim 36 \mathrm{~km} . \mathrm{s}^{-1}$, well matched to its expected intrinsic linewidth and more than adequate for dynamical studies.

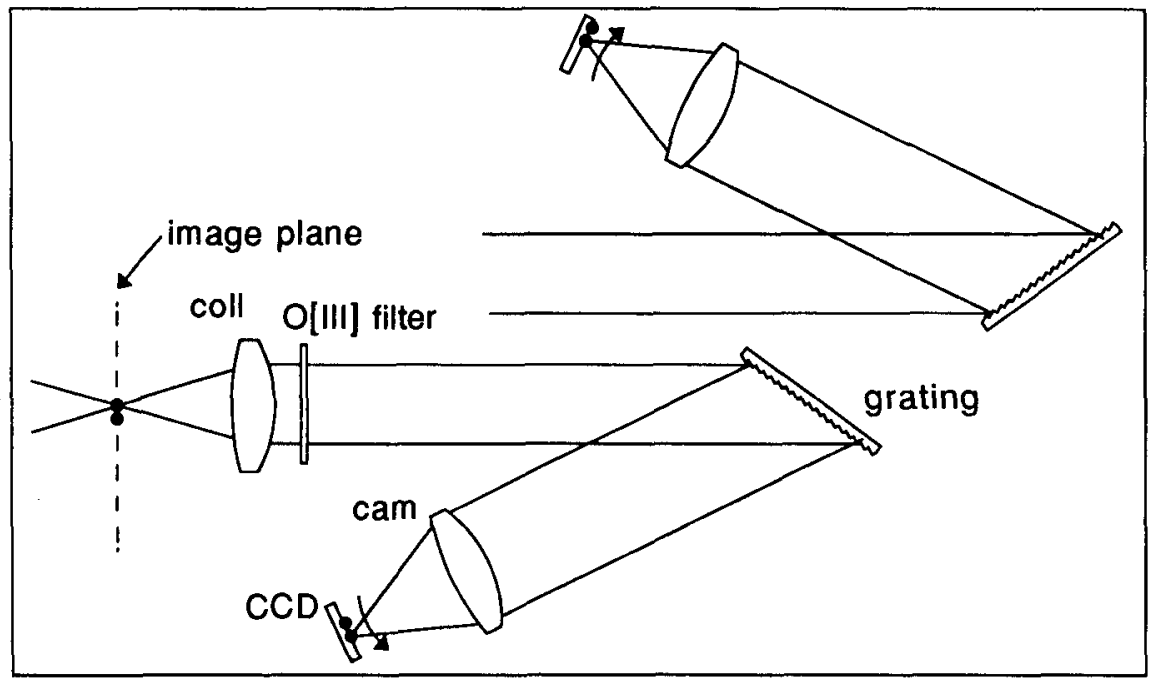

Figure 1. Slitless spectrograph in normal and rotated operation. The arrows show the dispersion direction, whereas the dots indicate points in the image.

Ford et al have demonstrated the similarity in the shape and high-luminosity cutoff of the PNe luminosity functions between local group members, M81, Cen $\mathbf{A}$ and members of the Leo group. For Cen $\mathbf{A}$ they obtained an absolute [OIII] luminosity function complete over a 1.6 magnitude range. The brightest $\mathrm{PNe}$ have a flux of $1.3 \times 10^{-15}$ ergs.s ${ }^{-1} . \mathrm{cm}^{-2}$ while (incomplete) detection extends over a $\geq 2.5$ magnitudes range. Ford et al also produced a PNe surface density measure for Cen $A$ of $1.14 \mathrm{PNe}$ per integrated $\mathrm{m}_{B}=15$ surface brightness within the completeness range, implying a total $\mathrm{PNe}$ count of 1161 down to an [OIII] flux level of $3 \times 10^{-16} \mathrm{ergs.s}^{-1} . \mathrm{cm}^{-2}$.

Using the slitless spectroscopy technique, the detection threshold can be extended producing either a larger number of detected $\mathrm{PNe}$ or a probe to greater distances. Given an on-source exposure time of 4 hours, an interference filter bandpass of $15 \AA$, seeing at the $1.5^{\prime \prime}$ level, and an efficiency of $4 \%$ characteristic of the AAT spectrograph example above, a $(\mathrm{S} / \mathrm{N}=5)$ flux limit of $4.5 \times 10^{-17}$ ergs. $\mathrm{s}^{-1} . \mathrm{cm}^{-2}$ could be achieved. For Cen $\mathrm{A}$ this would extend the detection threshold by $\geq 2$ magnitudes and produce an approximately linear increase in $\mathrm{PNe}$ yield. We have used models incorporating instrumental effects and realistic 
PNe surface density and luminosity functions in order to verify these estimates, to quantify the effects of galaxy background contamination and to refine the algorithms required to pick out the $\mathrm{PNe}$ from the continua at $\mathrm{S} / \mathrm{N} \leq 5$.

\section{A Planetary Nebulae seeker}

In 1959 Pierre Connes published an interesting method of splitting light into two beams (Revue $D^{\prime} O p t i q u e, 38, n^{\circ} 4,157-201$ ). It involves the use of a grating illuminated at normal incidence, arranged in such a way that light goes exclusively into the +1 and -1 orders, higher orders being excluded by the groove spacing. The instrument we have been considering in this paper could be constructed with such a beamsplitter, which would also serve as the dispersive element (see Fig 2).

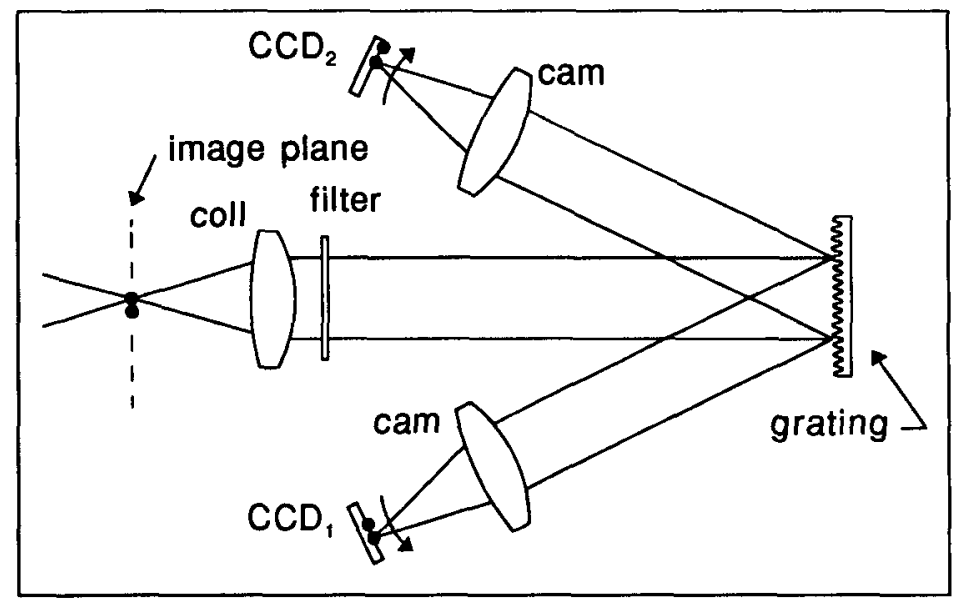

Figure 2. Schematic of the two-arm slitless spectrograph. The grating shown has a groove depth of $375 \mathrm{~nm}$ (see text).

We have chosen for this illustration not the blazed grating of Connes but a holographic grating. In each arm is placed a camera, complete with CCD. Point sources in the focal plane will be imaged antisymmetrically as shown, while as a dispersive element the grating works symmetrically so that the optical system has exactly the desired properties. Instead of rotating the spectrograph between two exposures, the configuration is fixed for the total integration time. Advantages include the absence of moving parts, bringing increased rigidity and reproducibility. Even more important is the simultaneity of the registration of the two images, nulling the effect of any changes in photometric conditions.

Quite possibly, the optical efficiency will be better than in the rotating spectrograph since, if the grating is designed to reject zeroth order, the total efficiency will probably be close to $100 \%$. This can be done by adjusting the groove depth to be an uneven multiple of $\lambda / 4$. For $\lambda=5007 \AA$ the grating shown will satisfy this condition. The operational efficiency will be higher than 
for the rotating spectrograph since, whatever total integration time is available, the signal will be equally divided between the two frames.

As an example, consider an $1800 \mathrm{gr} / \mathrm{mm}$ holographic grating whose surface is normal to the incident $150 \mathrm{~mm}$ collimated beam from a 4 metre telescope. The first order spectra, centred at $5007 \AA$, are formed at $64.32^{\circ}$ which, with a camera focal length of $400 \mathrm{~mm}$ and the same CCD, implies $0.46^{\prime \prime} / \mathrm{pixel}$ in the undispersed direction with the orthogonal anamorphism producing $0.20^{\prime \prime} / \mathrm{pixel}$ with $43 \mathrm{~km} \cdot \mathrm{s}^{-1} /$ ", well tuned to PNe characterisation.

\section{Conclusions}

A technique is sought which can quickly categorize all or most of the bright Planetary Nebulae in an external galaxy. Using a novel application of slitless spectroscopy the data can be obtained by one or two exposures with a single instrument, rather than the patchwork of procedures presently necessary. Calibration problems, which dominate the quality of existing data, can be significantly simplified.

The improved luminosity functions obtained, together with the systemic radial velocity, would be a powerful independent means of estimating the Hubble relation, while the radial velocity distribution of the PNe would be among the best available probes of the galaxy's spheroidal velocity field and hence of the mass distribution.

\section{Discussion}

A. Chrysostomou: How will you differentiate between other line emitting regions, such as compact $H$ II regions?

N. Douglas: You cannot. But elliptical galaxies, which are the main targets for such programs, will be dominated by PNe. H II regions might be good targets in late-type spirals, provided the $H$ II regions were point-like. Of course, for determining the rotation curves it doesn't matter what objects you pick up as long as they have the narrow emission line.

G. Comte: Can you comment on the applicability of the PNe seeker to the search for absorption-feature objects like e.g. carbon stars in more nearby galaxies?

$N$. Douglas: It would be totally inappropriate. An absorption feature in a single carbon star would be lost against the smeared-out continuum.

G. Monnet: Two short comments -

1. Your very interesting technique is a modern offspring of developments made a few kilometers from here, namely Charles Fehrenbach's 1947 objective prism, which was the same trick of two spectroscopic slitless exposures, rotated by $180^{\circ}$. 2. Holographic gratings with the desired property, i.e. basically all light in the -1 and +1 orders have been actually developed by Jobin-Yvon in the early 70 's.

N. Douglas: As I said, there's not too much which is truly new under the sun. We think though that our technique will be much more practical because we'll be able to handle the problem of aligning the two images much better. 